\title{
What's New in Prostate Cancer Research? Highlights of GU-ASCO 2015
}

Cite as: Can Urol Assoc J 2015;9(5-6-Supp|3):S148-53. http://dx.doi.org/10.5489/cuai.2994

Published online June 15, 2015.

T he 2015 Genitourinary Cancers Symposium (GU-ASCO 2015) was a forum for the presentation of new research in a number of tumour types, highlighting the most recent basic science and clinical work in the GU cancer field. The following pages summarize some of the most interesting new data and findings presented at this symposium.

\section{New research in early-stage prostate cancer}

\section{Risk associated with testicular cancer}

Investigators at the University of Maryland retrospectively analyzed the Surveillance, Epidemiology, and End Results (SEER) database to identify individuals with a history of testicular cancer and conduct a comparative analysis to determine whether or not these individuals were at higher risk for subsequent intermediate and high-risk prostate cancer (PCa) (Gleason score 7 or higher) compared to individuals with a history of melanoma. ${ }^{1}$

The researchers identified 32435 individuals with testicular cancer history and matched them to 147044 patients with a history of melanoma. Compared to those individuals with a history of melanoma, those with a history of testicular cancer had a 4.7-fold risk of developing any PCa and a 5.2fold risk of intermediate-high risk PCa (multivariate analyses, both $p<0.0001$ ). This retrospective data are provocative and hypothesis-generating.

\section{Caution on active surveillance for intermediate-risk PCa}

This Canadian study sought to determine the survival outcomes of patients with intermediate-risk PCa (prostate-specific antigen [PSA] $>10 \mathrm{ng} / \mathrm{ml}$ or Gleason score 7 or clinical stage T2b/2c). ${ }^{2}$ Study researchers identified 213 intermediate-risk patients managed with active surveillance from the single-centre database at Sunnybrook Health Sciences Centre in Toronto. These patients had a median follow-up of 6.7 years. A total of 732 low-risk patients managed with active surveillance were also included in the analysis as the control group.

Of the 213 intermediate-risk patients, 34.7\% received treatment (mainly radiation). Most of these patients were treated due to short PSA doubling times. The 10- and 15-year overall survival (OS) rates are shown in Figure 1. For each analysis, the survival rate was significantly lower for those patients in the intermediate-risk group.

\section{New research in metastatic, hormone-sensitive $\mathrm{PCa}$ ( $m H S P C)$}

\section{GETUG-AFU: No significant survival advantage for androgen-deprivation therapy (ADT) + docetaxel}

At GU-ASCO 2015, researchers presented an updated analysis of data from the Androgen-Deprivation Therapy Alone or with Docetaxel in Non-Castrate Metastatic Prostate Cancer (GETUG-AFU 15) study. ${ }^{3}$ This study included 385 patients with metastatic non-castrate PCa (mCRPC) and randomized them to treatment with ADT alone (luteinizing-hormone-releasing hormone [LHRH] agonist, maximum androgen blockade, or orchiectomy) or ADT with docetaxel $\left(75 \mathrm{mg} / \mathrm{m}^{2}\right.$ every three weeks for up to nine cycles). The primary endpoint was OS. To help facilitate a comparison with the previously reported ECOG 3805: ChemoHormonal Therapy Versus Androgen Ablation Randomized Trial for Extensive Disease in Prostate Cancer (CHAARTED) study, which stratified patients by high or low tumour volume, the investigators of the GETUG-AFU 15 study retrospectively assessed tumour volume and performed sub-analyses on these separate populations.

The median follow-up for this analysis was 82.9 months. Over this time, the median OS was 46.5 months for ADT alone and 60.9 months for ADT plus docetaxel. This numerical difference was not, however, statistically significant (HR 0.9, $95 \%$ confidence interval $[\mathrm{Cl}] 0.7$ to $1.2 ; p=0.44$ ). There were significant differences noted in secondary endpoints. Median biological progression-free survival (PFS) was 12.9 months with ADT alone and 22.9 months with ADT plus docetaxel (HR 0.7, 95\% Cl 0.6-0.9, $p=0.0021$ ).

For patients with high-volume disease $(n=183)$, median OS was 35.1 months for ADT alone and 39 months for ADT plus docetaxel (HR 0.8, 95\% Cl 0.6-1.2; $p=0.35)$. For low-volume disease, median OS was not reached for ADT alone and was 83.1 months for ADT plus docetaxel (HR 1.0, 95\% Cl 0.6-15, $p=0.87$.

The results of this trial stand in contrast to those of the similarly designed E3805 trial, which did report a survival benefit 


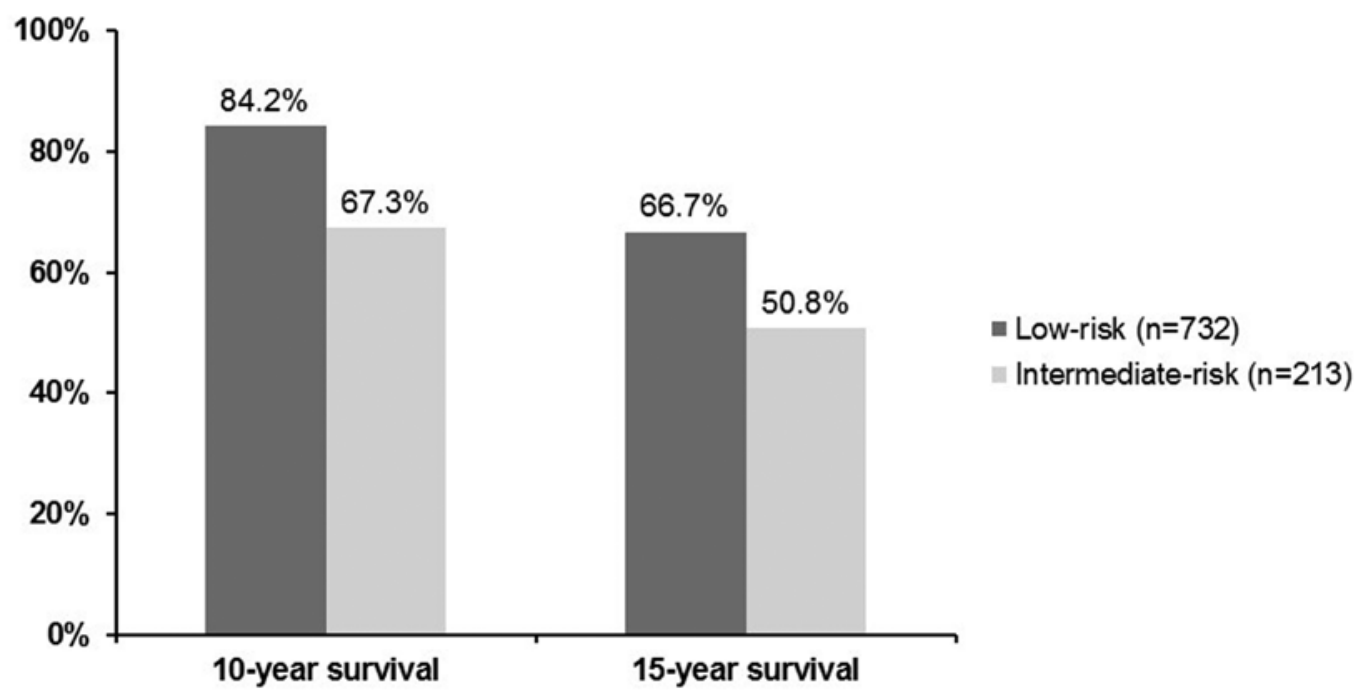

Hazard ratio (HR) for OS: $2.13, p<0.0001$.

Fig. 1. 10- and 15-year overall survival rates with active surveillance: Low-risk and intermediate-risk patients.

for ADT plus docetaxel in high-volume disease (HVD) patients. ${ }^{4}$ At GU-ASCO 2015, Dr. Eric Small led a discussion session that included an assessment of the different outcomes of these two studies. ${ }^{5}$ He postulated that the difference between the two studies is likely explained by a few important differences in the trial designs and populations (Table 1).

The GETUG-AFU 15 trial was underpowered to detect differences in the high-volume group $(\mathrm{N}=183)$. Of potential importance was the median time of follow-up, namely 83 months for GETUG-AFU 15 and 29 months for ECOG 3805. The nonprotocol treatments received differed as well. In GETUG-AFU $15,80 \%$ of those in the ADT-only arm and $45 \%$ of the ADT plus docetaxel arm received post-protocol docetaxel; in the ECOG study, $33 \%$ of the ADT-only arm and $12 \%$ of the ADT plus docetaxel arm had received post-protocol docetaxel.

Finally, the use of oral agents (abiraterone or enzalutamide) was not reported in the GETUG-AFU 15 trial, but is thought to be low, whereas these agents were used post-protocol in $20 \%$ of the ECOG 3805 study's ADT-only arm and $23 \%$ of the ADT plus docetaxel arm.

\section{Abiraterone in $\mathrm{mHSPC}$}

The Southwest Oncology Group (SWOG) S10104 study was a Phase 2, single-arm analysis of abiraterone acetate plus prednisone among 41 men with poor prognosis metastatic disease (PSA $>4 \mathrm{ng} / \mathrm{ml}$ after ADT). ${ }^{6}$ The primary endpoint was a PSA of $\leq 0.2 \mathrm{ng} / \mathrm{mL}$ within one year. Of the 41 patients, five (13\%) achieved this goal, thereby falling short of the protocol-specified level of six responses; however, the authors were nonetheless encouraged. The median OS noted in this population was 23.9 months, which is substantially longer than that observed in a similar population of patients (SWOG 9346 study, 13 months ${ }^{7}$ ).

Table 1. Key differences between GETUG-AFU 15 and ECOG 3805 studies

\begin{tabular}{lcc} 
& GETUG \\
\hline Total sample size & 385 & 790 \\
High-volume disease & $183(47.5 \%)$ & $514(65 \%)$ \\
Median follow-up & 83 months & 29 months \\
Received post-protocol docetaxel & ADT-only arm: $80 \%$ & ADT-only arm: $33 \%$ \\
& ADT-docetaxel arm: $45 \%$ & ADT-docetaxel arm: $12 \%$ \\
Received post-protocol abiraterone or enzalutamide & ADT-only arm: No response & ADT-only arm: $20 \%$ \\
& ADT-docetaxel arm: No response & ADT-docetaxel arm: $23 \%$
\end{tabular}

GETUG-AFU: Groupe d'Etude des Tumeurs Uro-Genital-Association Française d'Urologie; ECOG: Eastern Cooperative Oncology Group; ADT: androgen deprivation therapy. 


\section{New Research in mCRPC}

\section{No OS benefit of cabozantinib among men with prior docetaxel and abir-} aterone or enzalutamide

The Study of Cabozantinib (XL184) Versus Prednisone in Men With Metastatic Castration-resistant Prostate Cancer Previously Treated With Docetaxel and Abiraterone or MDV3100 (COMET-1) study was an international, Phase 3 trial involving 960 patients post-progression with docetaxel and post-progression with abiraterone and/or enzalutamide. ${ }^{8}$ All patients had bone metastases, with or without severe pain. Patients were randomized to receive oral cabozantinib $60 \mathrm{mg}$ daily $(\mathrm{n}=682)$ or prednisone $5 \mathrm{mg}$ twice daily $(\mathrm{n}=346)$. The primary study endpoint was OS.

For the primary endpoint analysis, there was no significant difference between the groups. The median was 11.0 months for cabozantinib and 9.8 months with prednisone $(p=0.213$; Fig. 2). In subgroup analyses, the only group who showed a significant difference in favor of cabozantinib were those who had previously been treated with cabazitaxel $(n=393)$.

With respect to secondary endpoints, there were some benefits observed with cabozantinib. The proportion with a bone scan response at week $12(\geq 30 \%$ reduction in bone scan lesion area by central review) was $42 \%$ with cabozantinib and $3 \%$ with prednisone $(p<0.001)$. Bone biomarkers were also improved relative from baseline. Median PFS was also longer with cabozantinib (5.6 months) versus with prednisone $(2.8$ months) $(p<0.001)$.

In the analysis of safety, $33 \%$ of those in the cabozantinib group discontinued the study due to adverse events, compared to $12 \%$ of the prednisone group.

\section{Enzalutamide safe and effective in older men (sub-analysis)}

Researchers from the Safety and Efficacy Study of Oral MDV3100 in Chemotherapy-Naive Patients With Progressive Metastatic Prostate Cancer (PREVAIL) study group presented an analysis of efficacy and safety results from this study for the subgroups of patients younger than 75 years $(n=1108)$ or 75 years and older $(n=609) .{ }^{9}$ The PREVAIL study was a randomized, controlled clinical trial comparing oral enzalutamide to placebo among 1,717 chemotherapy-naïve men with mCRPC. The study showed that enzalutamide treatment was associated with significant improvements in OS and radiographic PFS compared to placebo in these patients. Those patients over 75 years had a slightly higher incidence of decreased appetite, asthenia and falls.

In this sub-analysis, the investigators reported that patients in the older subgroup had worse prognostic features at baseline relative to the younger patients (e.g., higher proportion with ECOG performance status grade 1, higher median PSA, higher proportion with $>20$ bone metastases and higher prevalence of

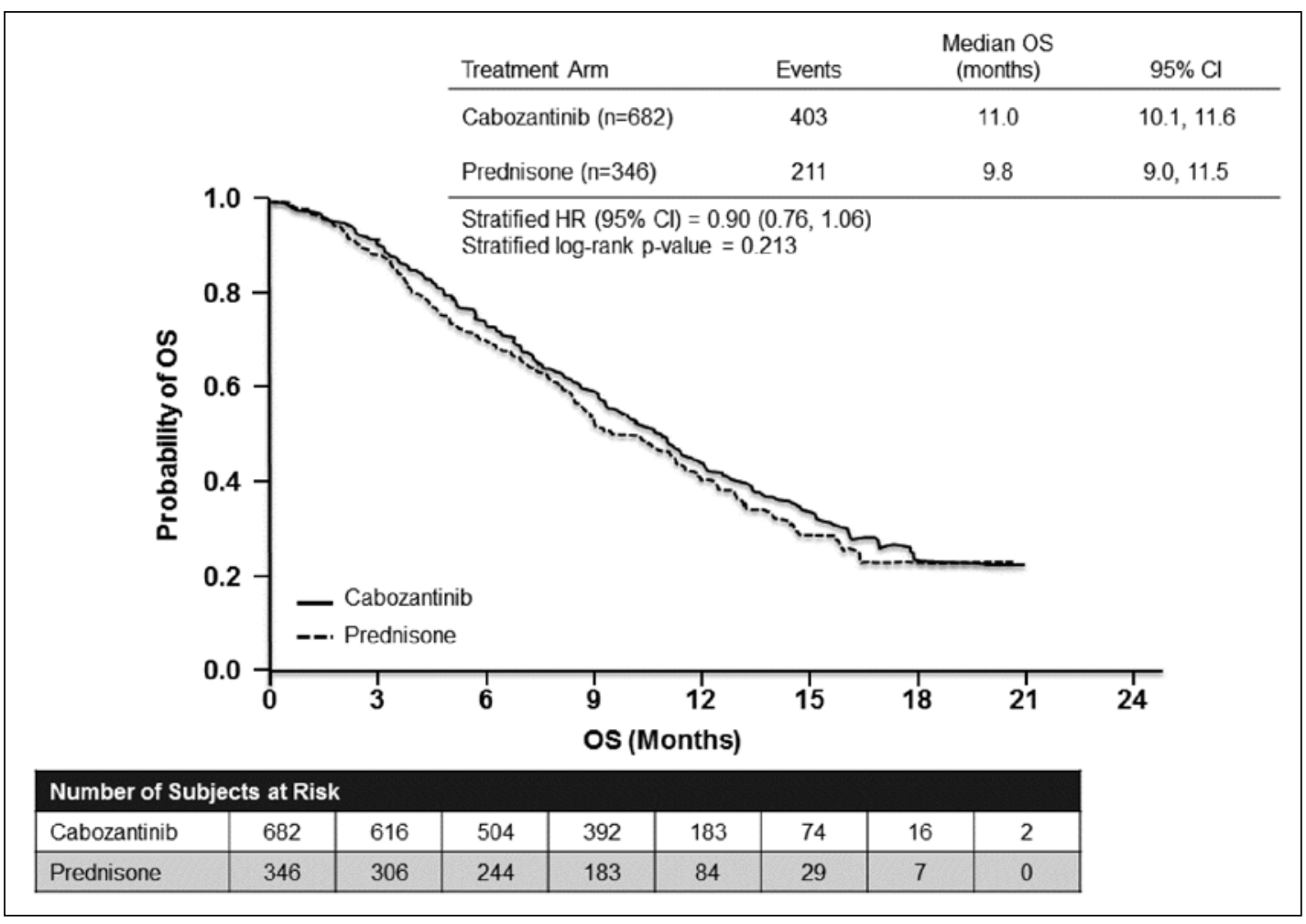

Fig. 2. Cabozantinib after progression on docetaxel and abiraterone or enzalutamide (COMET-1): Overall survival. 
cardiovascular disease). Enzalutamide treatment was associated with significant risk reductions for death of $40 \%$ in the older subgroup and $23 \%$ in the younger group (both statistically significant vs. placebo). Radiographic PFS was reduced by $83 \%$ with enzalutamide in the older subgroup and $80 \%$ among the younger patients (Fig. 3).

\section{Some new insight on sequencing of therapies}

A post-hoc analysis of data from the Abiraterone Acetate in Asymptomatic or Mildly Symptomatic Patients With Metastatic Castration-Resistant Prostate Cancer (COU-AA-302) trial showed that taxane treatment after progression on abiraterone acetate plus prednisone is a viable strategy. ${ }^{10}$ The median time to PSA progression on subsequent taxane therapy was 7.59 months in this analysis.

Similarly, in a post-hoc analysis of data from a subset of patients from the PREVAIL study showed that taxanes retain their efficacy among patients pre-treated with enzalutamide. ${ }^{11}$

\section{Impact of AR-V7 splice variant on treatment efficacy}

Previous research had shown that the AR-V7 splice variant was associated with primary resistance to both abiraterone acetate and enzalutamide. At GU-ASCO 2015, researchers presented data on 37 men who initiated therapy with a taxane; 17 of these patient had the AR-V7 splice variant confirmed. ${ }^{12}$ The study showed that the presence of AR-V7 was not associated with resistance to taxane therapy. The authors suggested that this could be used as a biomarker to help guide the selection of therapy among patient with mCRPC.
Also of interest in the AR-V7 discussion is the upcoming Androgen Receptor Modulation Optimized for Response in Splice Variant (ARMOR3-SV) trial comparing enzalutamide and the small molecule galeterone among patients with the AR-V7 variant. ${ }^{13}$ This randomized, open-label, Phase 2 trial is expected to begin recruiting in 2015 .

\section{New evidence with radium-223}

At GU-ASCO 2015, the evidence continued to accumulate for radium-223 in the mCRPC setting. This included two real-world analyses from the expanded access program (EAP) in the United States. ${ }^{14-16}$ The EAP includes 184 patients with symptomatic bone metastases, two or more skeletal metastases on imaging, and no lung, liver, or brain metastases, treated with radium-223 $50 \mathrm{kBq} / \mathrm{kg}$ every four weeks for six injections (in addition to best standard of care). The primary endpoint of the study is safety.

Analysis of these patients did not reveal any new safety concerns, and radium-223 was well tolerated overall ${ }^{14}$ and among those patients concurrently receiving abiraterone acetate or enzalutamide. ${ }^{15}$ Median OS, one of the key exploratory efficacy analyses, was 17 months. ${ }^{14}$ The impact of radium-223 on pain was significant; $43 \%$ of patients reported pain relief on the Brief Pain Inventory (Short Form), while 19\% reported no change and $28 \%$ reported worsening of pain. ${ }^{16}$

Also presented at GU-ASCO 2015 was a study of 46 patients with $\mathrm{mCRPC}$ showing that the addition of radium-223 to docetaxel therapy was associated with a greater proportion of patients achieving normalization of bone alkaline phosphatase (bALP), a bone turnover marker, compared to docetaxel alone. ${ }^{17}$

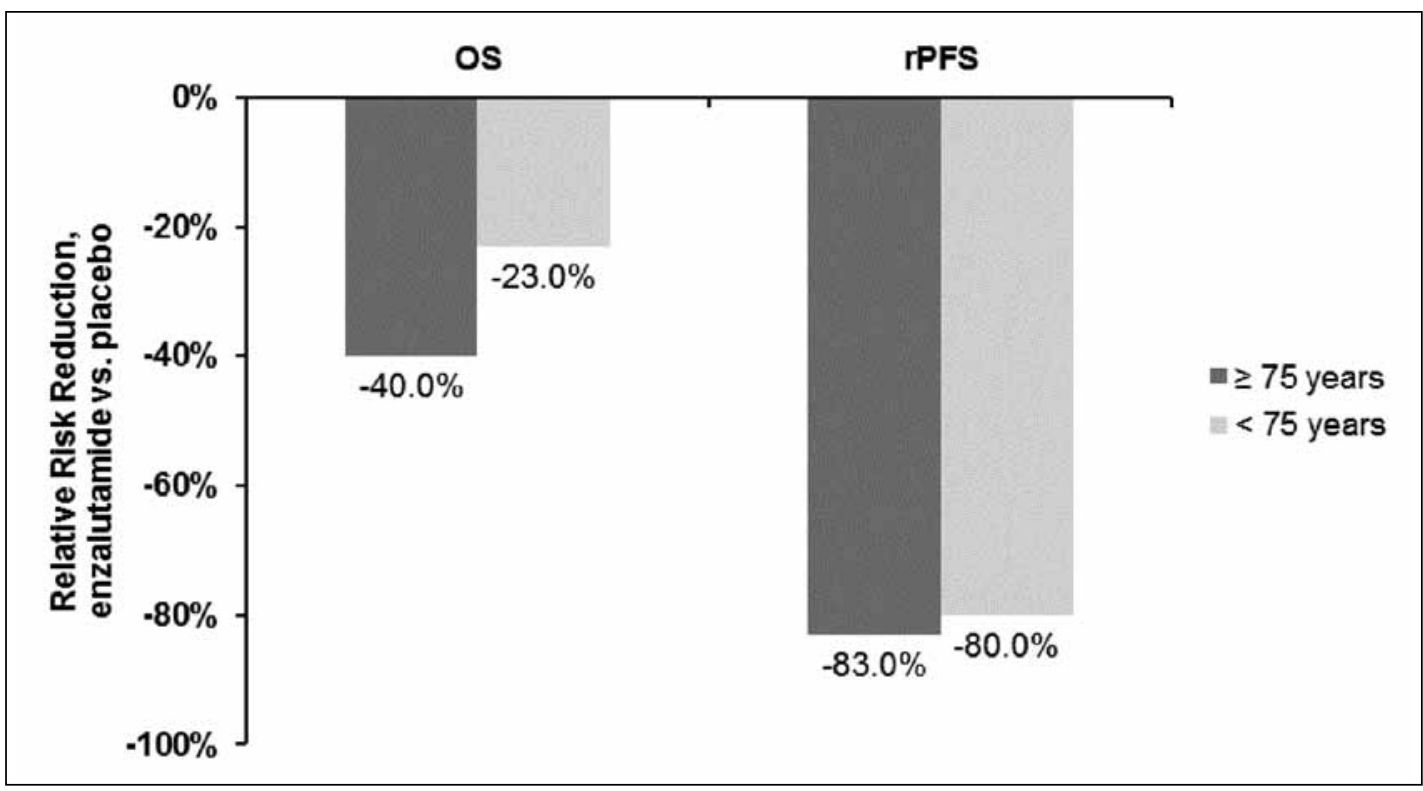

Fig. 3. Overall and radiographic progression-free survival in the PREVAIL study (enzalutamide vs. placebo) stratified by age. 


\section{ODM-201, a promising novel therapy for mCRPC}

With respect to novel therapies in development for $\mathrm{mCRPC}$, one of the more promising compounds appears to be ODM-201, an oral AR-inhibitor. In an open-label Phase 1 study, this compound was well tolerated and showed substantial anti-tumour activity. ${ }^{18}$ The Phase 3 Efficacy and Safety Study of ODM-201 in Men With High-risk Non-metastatic Castration-resistant Prostate Cancer (ARAMIS) is ongoing.

\section{New research in radiation therapy}

\section{No OS benefit from dose escalation in intermediate-risk patients}

Results of the Radiation Therapy in Treating Patients With Stage II Prostate Cancer (RTOG 0126) trial were presented at GU-ASCO 2015. This was a Phase 3, randomized study of highdose three-dimensional conformal radiation therapy (3DCRT)/ intensity-modulated radiation therapy (IMRT) versus standard dose in 1,532 men with localized PCa, stage cT1b-T2b with Gleason Score 2 to 6 and PSA $\geq 10$ and $<20 .{ }^{19}$ The study treatments were 3DCRT or IMRT to 79.2 Gy in 44 fractions (highdose) or 70.2 Gy in 39 fractions (standard dose). The primary endpoint was OS.

There was no significant difference in OS (HR 0.98, 95\% Cl 0.79-1.21, $p=0.87$ ) (Fig. 4); however, there were significant improvements noted in favour of the high-dose group for local control, distant-metastasis-free survival, and biochemical dis- ease failure rates, as well as less use of salvage therapies. These benefits were offset by a significant increase in gastrointestinal and GU toxicity than the standard dose.

\section{Additional benefit with short-term ADT with radiotherapy in intermediate- risk patients}

A Canadian study presented at GU-ASCO evaluated the impact of short-term ADT (STADT) among patients with intermediaterisk $\mathrm{PCa}$ treated with radiotherapy..$^{20}$ In the Study on the Role of Hormonal Treatment for Two Dosage Levels of Prostate Radiation Therapy Versus Prostate Radiation Therapy Alone (PCS III), patients were randomized to receive STADT in combination with either a 70 Gy or 76 Gy dose of radiotherapy, or with radiation alone (76 Gy). The co-primary endpoints were biochemical failure and disease-free survival.

After a median follow-up of 75.4 months, there was a significant benefit for both co-primary endpoints in favour of the inclusion of STADT compared to radiotherapy alone. There was not significant between-group difference, however, with respect to OS (secondary endpoint).

\section{Low-dose-rate brachytherapy is superior to dose-escalated external beam radiation in unfavorable-risk $\mathrm{PCa}$}

The Androgen Suppression Combined With Elective Nodal and Dose Escalated Radiation Therapy (ASCENDE-RT) study included 122 patients with intermediate- and high-risk PCa. ${ }^{21}$ All patients received one year of ADT with an LHRH agonist, plus a non-steroidal anti-androgen for at least one month. After

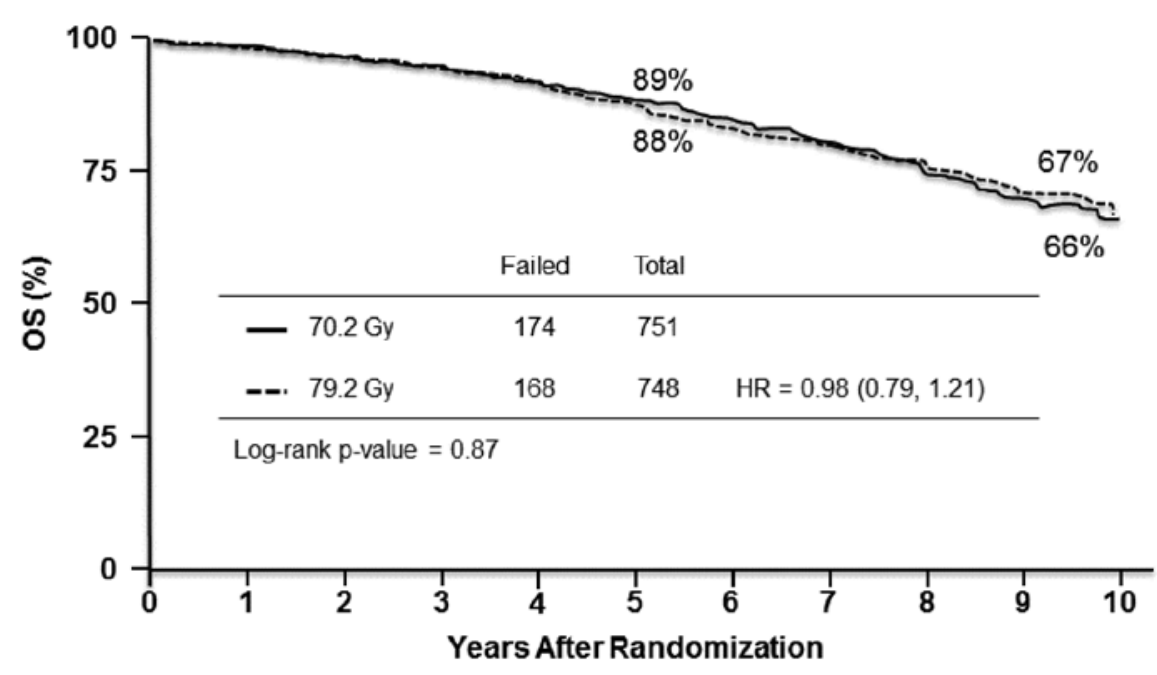

\begin{tabular}{|l|l|l|l|l|l|l|l|l|l|l|l|}
\hline Patients at Risk \\
\hline $70.2 \mathrm{~Gy}$ & 751 & 735 & 708 & 688 & 660 & 616 & 515 & 377 & 241 & 128 & 53 \\
\hline $79.2 \mathrm{~Gy}$ & 748 & 729 & 708 & 683 & 648 & 602 & 492 & 379 & 243 & 122 & 52 \\
\hline
\end{tabular}

Fig. 4. High-dose versus standard dose radiotherapy for localized prostate cancer: Overall survival. 
eight months, all patients received external-beam, whole-pelvis radiation therapy (EBRT; 46 Gy in 23 fractions). Subjects were randomized to treatment with dose-escalated EBRT (EBRT-B; boost of 32 Gy in 16 fractions) or low-dose-rate brachytherapy (LDR-B; lodine-125 LDR boost prescribed to a minimum peripheral dose of 115Gy). The primary study endpoint was relapse-free survival, defined by biochemical criteria.

Over a median follow-up of 6.5 years, LDR-PB was associated with a $50 \%$ reduction in biochemical relapse compared to EBRT-B; however, there was no significant between-group difference in OS (or in PCa-specific survival or metastasis-free survival).

\section{Insights in imaging for prostate cancer}

One of the recognized shortcomings of the PCa field is the inadequacy of current imaging modalities as means of monitoring for emerging metastases. At GU-ASCO 2015, Dr. Peter Pinto presented an overview of recent research with novel imaging techniques. $^{22}$

Perhaps the most promising modality he discussed was fluoride positron emission tomography-computed tomography (PET/ CT) scanning, which has been shown to be superior to technetium (TC) bone scan. Ferumoxytol (an iron-based compound)enhanced magnetic resonance imaging (MRI) may also be useful for lymph node imaging. F-FDHT is an androgen analog with high affinity for the androgen receptor. This has promise as a non-invasive way to monitor treatment response.

Modalities that have not proven to be useful include choline PET or acetate/PET for low PSA metastases. Prostate-specific membrane antigen (PSMA), a transmembrane protein found in the prostate, has been shown to be more specific than either choline or acetate PET.

\section{References}

1. Riggin J, Siddiqui MM. Development of intermediate and high-risk prostate cancer after testicular cancer. J Clin Oncol 2015; 33(suppl 7; abstr 177).

2. Musunuru HB et al. Cautionary tale of active surveillance in intermediate-risk patients: Overall and cause-specific survival in the Sunnybrook experience. J Clin Oncol 2015; 33(suppl 7; abstr 163).

3. Gravis $G$ et al. Androgen deprivation therapy (ADT) plus docetaxel (D) versus ADT alone for hormone-naive metastatic prostate cancer (PCa): Long-term analysis of the GETUG-AFU 15 phase III trial. J Clin Oncol 2015; 33(suppl 7; abstr 140).
4. Sweeney $C$ et al. Impact on overall survival (OS) with chemohormonal therapy versus hormonal therapy for hormone-sensitive newly metastatic prostate cancer (mPrCa): An ECOG-led phase III randomized trial. J Clin Oncol 2014; 32:5s(suppl; abstr LBA2).

5. Small EJ. Looking in the crystal ball or could the negative outcomes of these phase 3 trials been predicted? Discussion presented at GU-ASCO 2015; Abstract Discussion.

6. Flaig TW et al. Phase II trial of abiraterone acetate (AA) treatment for metastatic prostate cancer (PC) patients with a PSA of more than four following initial androgen deprivation therapy: SWOG S1014. J Clin Oncol 2015; 33 (suppl 7; abstr 152)

7. Hussain $M$ et al. Absolute prostate-specific antigen value after androgen deprivation is a strong independent predictor of survival in new metastatic prostate cancer: data from Southwest Oncology Group Trial 9346 (INT0162). J Clin Oncol 2006; 24(24):3984-90. http://dx.doi.org/10.1200/JC0.2006.06.4246

8. Smith MR et al. Final analysis of COMET-1: Cabozantinib (Cabo) versus prednisone (Pred) in metastatic castration-resistant prostate cancer (mCRPC) patients (pts) previously treated with docetaxel (D) and abiraterone (A) and/or enzalutamide (E). J Clin Oncol 2015; 33(suppl 7; abstr 139).

9. Graff JN et al. Clinical outcomes and safety in men $\geq 75$ and $<75$ years with metastatic castration-resistant prostate cancer (mCRPC) treated with enzalutamide in the phase 3 PREVAlL trial. J Clin Oncol 2015; 33(suppl 7; abstr 200)

10. De Bono IS et al. Response to taxane chemotherapy as first subsequent therapy after abiraterone acetate (AA) in patients (pts) with metastatic castration-resistant prostate cancer (m(RPC): Post-hoc analysis of COU-AA-302. J Clin Oncol 2015; 33(suppl 7; abstr 184).

11. Gizzi $M$ et al. Previous enzalutamide therapy and response to subsequent taxane therapy in metastatic castrationresistant prostate cancer. J Clin Oncol 2015; 33(suppl 7; abstr 227).

12. Antonarakis ES et al. AR splice variant 7 (AR-V7) and response to taxanes in men with metastatic castrationresistant prostate cancer (mCRPC). J Clin Oncol 2015; 33(suppl 7; abstr 138).

13. Roberts $」$ et al. Androgen receptor modulation optimized for response in splice variant (ARMOR3-SV): Randomized, open-label, multicenter, controlled study of galeterone versus enzalutamide (enz) in men expressing AR-V7 splice variant (SV), metastatic castrate resistant prostate cancer (m(RPC). J Clin Oncol 2015; 33(suppl 7; abstr 259).

14. Vogelzang NJ et al. Radium-223 dichloride (Ra-223) in U.S. expanded access program (EAP). J Clin Oncol 2015; 33(suppl 7; abstr 247).

15. Sartor $\mathrm{A} 0$ et al. Prior and concurrent use of abiraterone and enzalutamide with Ra-223 in an expanded access setting. J Clin Oncol 2015; 33(suppl 7; abstr 253)

16. Morris MJ et al. Effect of radium-223 dichloride (Ra-223) on pain from US EAP. J Clin Oncol 2015; 33(suppl 7; abstr 160).

17. Morris MJ et al. Effects of radium-223 dichloride (Ra-223) with docetaxel (D) versus D on prostate-specific antigen (PSA) and bone alkaline phosphatase (bALP) in patients (pts) with castration-resistant prostate cancer (CRPC) and bone metastases (mets): A phase 1/2a clinical trial. J Clin Oncol 2015; 33(suppl 7; abstr 202).

18. Massard C et al. Pharmacokinetics, activity, and safety of ODM-201 in chemotherapy-naive patients with metastatic castration-resistant prostate cancer: An open-label phase I trial with long-term extension. J Clin Oncol 2015; 33(suppl 7; abstr 230)

19. Michalski JM et al. A randomized trial of 79.2Gy versus 70.26 y radiation therapy (RT) for localized prostate cancer. J Clin Oncol 2015; 33(suppl 7; abstr 4).

20. Nabid A et al. Place of short-term androgen deprivation therapy in intermediate-risk prostate cancer treated with radiotherapy: a phase III trial. J Clin Oncol 2015; 33(suppl 7; abstr 5).

21. Morris WJ et al. ASCENDE-RT: A multicenter, randomized trial of dose-escalated external beam radiation therapy (EBRT-B) versus low-dose-rate brachytherapy (LDR-B) for men with unfavorable-risk localized prostate cancer. $J$ Clin Oncol 2015; 33(suppl 7; abstr 3)

22. Pinto PA. New imaging modalities to identify early metastatic disease. Presentation at the at GU-ASCO 2015; General Session 2. 